\title{
EQUIPO DE CLIMATIZACIÓN TIPO CENTRAL CON ADAPTACIONES PEDAGÓGICAS EN CLASES EXPERIMENTALES DE TERMODINÁMICA
}

MSc. Marta G. STOPPELLO, Lic. Guillermo R. SÁNCHEZ y Dr. Arturo J. BUSSO (*)

\section{RESUMEN}

E1 Grupo en Energías Renovables (GER) de la Facultad de Ciencias Exactas Naturales y Agrimensura - FaCENAimplementó el proyecto "Desarrollo de equipos didácticos para el fortalecimiento de la enseñanza de la Física”. Para fortificar la formación de ingenieros eléctricos, en eventos físicos reales, ha modificado con fines pedagógicos un equipo convencional de climatización tipo central, instalado en el Laboratorio de Termodinámica del Departamento de Física. Su aplicación didáctica es en el dictado de Trabajos Prácticos de laboratorio en Termodinámica en la carrera Ingeniería Eléctrica. En el montaje las modificaciones corresponden a la incorporación de manómetros en los lados de alta y baja presión con válvula de servicio, sensores de temperatura en los tubos de conexión de la unidad condensadora y evaporadora, y un control electrónico de la unidad con posibilidad de adquisición de datos. Aquí se presenta la descripción de las innovaciones y un ejemplo de trabajo experimental realizado por alumnos en los cursados de 2016 y 2017.

\section{ABSTRACT}

The Group on Renewable Energies (GER) of the Faculty of Natural Sciences and Surveying -FaCENA - implemented the project "Development of teching equipment for strengthening the education in physics". In order to fortify the formation of electrical engineers, in real physical events, a conventional central type air conditioning equipment installed in the Laboratory of Thermodynamics of the Department of Physics has been modified for pedagogical purposes. This equipment is used for teaching purposes within the laboratory practices included in the course of Thermodynamics in the Electrical Engineering career. In the assembly the modifications correspond to the incorporation of pressure gauges in the high and low pressure sides with service valve, temperature sensors in the connecting pipes of the condensing and evaporator unit, and the electronic control of the unit with possibility of data acquisition. In what follows the description of the innovations and an example of experimental work done by students in the course of 2016 and 2017 is presented.

(*) Grupo en Energías Renovables Facultad de Ciencias Exactas y Naturales y Agrimensura, Universidad Nacional del Nordeste. Av. Libertad 5470. Corrientes.e-mail: mstopello@hotmail.com 
Palabras clave: equipo frigorífico, climatización central, equipo adaptado, perspectiva situada.

\section{INTRODUCCIÓN}

Una idea aceptada es lo complejo y costoso de los equipos que se emplean en la ingeniería, situación que se hace más grave en lo relativo a la utilización didáctica de los mismos, puesto que son muy pocas las empresas que a nivel mundial fabrican equipos que puedan ser usados en la enseñanza de las Ingenierías.

La relación teoría y práctica sería la distancia que los graduados perciben entre los contenidos desarrollados durante sus trayectorias como estudiantes y las características que asumen las tareas cotidianas en el desempeño del rol. Desde esta óptica, fomentar la articulación entre aspectos teóricos y prácticos supone la necesidad de "tender puentes” entre la formación académica adquirida y las características del quehacer profesional (De Formento 2009, en [1]).

El desafío para la educación se orienta hacia la propuesta de secuencias didácticas cuya resolución requiera por parte del alumno alguna inserción concreta en contextos genuinos de desempeño del rol: desarrollar habilidades y conocimientos propios de la profesión, enfrentarse a problemas y situaciones reales. [2].

Si bien la práctica se plantea como fundamental para la formación de los profesionales de la Ingeniería, ésta se articula directamente con la teoría. El desarrollar actividades prácticas plantea reflexionar sobre lo que se trata de hacer, y por tanto, teorizar sobre ello (De Carr, 1996, en [2]).

Es necesario que el alumno se familiarice con un entorno muy parecido al laboral, por ello, se han desarrollado equipos didácticos con tecnología propia, realizados por los profesores con estudiantes, éstos últimos como parte de sus actividades de adscripciones. Se diseñaron y construyeron algunos equipos con el propósito de ser usados como herramientas de trabajo para la realización de prácticos experimentales y al mismo tiempo que su propia construcción sea un mecanismo didáctico. Los alumnos se involucran desde las actividades preparatorias de los ensayos, familiarizándose con el sistema de adquisición de datos y montado de los sensores en los puntos de medición. Así mismo, realizan la bajada de datos recolectados y el procesamiento de los mismos. Esta modalidad de trabajo hace que surjan cuestionamientos, tanto técnicos como teóricos en los temas abordados. Las actividades que se proponen se orientan a que el laboratorio sea un espacio de construcción colectiva y de trabajo en equipo; superando las visiones que hacen del laboratorio un ámbito de mediciones acríticas y manipulación de instrumentos [3]. Esto es el resultado del trabajo de un equipo de profesionales de distintos campos de investigación de la educación y de la investigación experimental, cuyos aportes concurren en la materialización de equipos para la enseñanza experimental de las ingenierías. En este trabajo se presenta la descripción de la adaptación pedagógica de un equipo de climatización tipo central, con la característica 
de "caja abierta”, que muestra los elementos constituyentes y resultados de su aplicación en clases experimentales.

Se han consultado experiencias en otras unidades académicas: Grado en Ingeniería Eléctrica de la Universidad de Valladolid en la asignatura Termodinámica Técnica y Transmisión del calor [4]. Ingeniería Industrial, Mecánica y Pesquera de la Universidad Nacional Experimental Francisco de Miranda asignaturas Laboratorio de conversión de energía y Laboratorio de termodinámica aplicada. [5]. Ingeniería Mecánica y Producción Industrial de la Universidad de la República de Uruguay en las asignaturas motores de combustión, Transferencia de calor I y II y energía I y II. [6].

\section{METODOLOGÍA}

A partir de 2 (dos) aire acondicionado tipo central, cuyo destino primario era baja de servicios, se procedió a su reparación y montaje en el Laboratorio de Termodinámica con los siguientes objetivos:

a) Climatizar convenientemente el laboratorio.

b) Utilizar el equipamiento con fines didácticos

El Montaje realizado, a los efectos de cumplir ambos objetivos, fue: disponer los equipos en los extremos del Laboratorio (casi simétricos), y las unidades condensadores y evaporadoras solidarias, con pared de por medio.

Uno de los Equipos, también climatiza oficinas contiguas al Laboratorio, tal como lo ilustra la figura1.

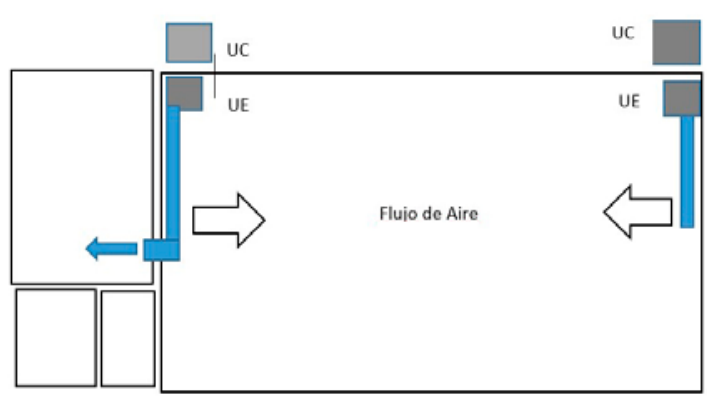

Figura 1: Ubicación unidades condensadoras y evaporadoras.

La disposición de Unidad Evaporadora (UE) y Unidad Condensadora (UC) próximas, permite un mejor registro y control de los parámetros eléctricos y termodinámicos. Un tablero de comandos controla los parámetros eléctricos. Un Rack de manómetros y válvulas de servicio, controla la carga y presiones del sistema. El esquema general se observa en la figura 2.

El dispositivo, posibilita múltiples aplicaciones:

a- Determinar coeficiente de desempeño de los ciclos de refrigeración, en forma puntual y dinámica.

b- Determinar coeficientes de desempeño de los ciclos de calefacción (Bombas de Calor), en forma puntual y dinámica.

c- Estudios de Psicrometría y confort humano.

d- Procesos de automatización y eficiencia energética.

e- Procesos de monitoreo y comando de equipos vía web.

f- Estudios de procesos físicos puntuales: Procesos isoentálpicos, isoentrópicos, cambios de fase y transmisión del calor. 


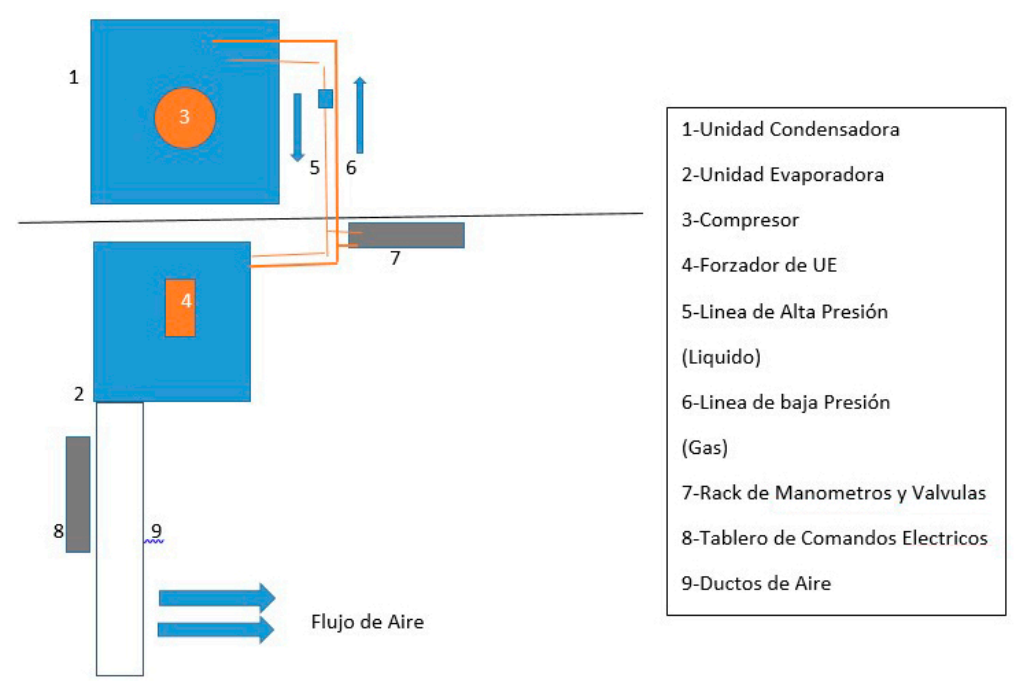

Figura 2: unidades condensadores y evaporadoras próximas

Para poder caracterizar el ciclo Frigorífico se requiere también, obtener temperaturas de distintos puntos del circuito, los cuales se realizan utilizando distintos tipos de sensores (termocuplas, termistores, diodos de silicio. etc.).

Para el desarrollo experimental se instalaron los siguientes equipos:

- Tablero para control de los parámetros eléctricos:

o Llave termomagnética tetrapolar

o Interruptores termomagnéticos para unidades interiores y exteriores.

o Contadores.

- Placa para automatizar el módulo: comando de bobinas de los contactores. (permite controlar el equipo en forma remota).

- Sensores de temperaturas: termocuplas, termistores y diodos de silicio. Puntos del circuito donde se instalaron los sensores:

o entrada y salida del comprensor o entrada y salida del evaporador

o entrada y salida del condensador

o salida del forzador

o ambiente

- Sensores de humedad: entrada y salida de flujo

- Rack de manómetros y válvulas de servicio para controlar la carga y las presiones del sistema.

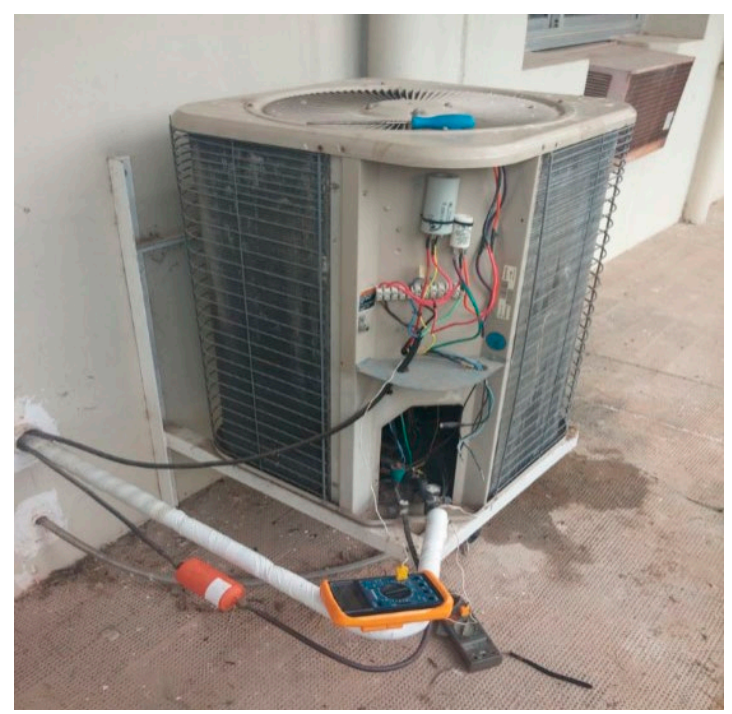

Figura 3: Fotografía de uno de los equipos 


\section{III: RESULTADOS Y DISCUSIÓN}

\section{Trabajo Práctico Experimental}

Objetivo general: Analizar el comportamiento de los ciclos termodinámicos de sistemas de refrigeración por compresión de vapor con el refrigerante R22.

\section{Objetivos Especificos:}

o Describir los diferentes dispositivos termodinámicos que componen a los ciclos de refrigeración.

o Obtener el COP de un ciclo simple de compresión de vapor con el refrigerante R22.

o Obtener el COP del equipo por transferencia de calor del flujo de aire del sistema

El trabajo práctico fue abordado en forma integral, por varios grupos de ternas, evaluando variación del COP, consumo energético, temperatura y humedad del recinto; todos en función del tiempo.

El ciclo de refrigeración que se utiliza con mayor frecuencia es el de refrigeración por compresión de vapor, en el que intervienen cuatro componentes principales:

- Compresor: el refrigerante llega en estado gaseoso al compresor y aumenta su presión.

- Condensador: es un intercambiador de calor.

- Sistema de expansión: el refrigerante líquido entra en el dispositivo de expansión donde reduce bruscamente su presión, con lo cual reduce su temperatura.

Evaporador: el refrigerante a baja tem- peratura y presión pasa por el evaporador, que al igual que el condensador es un intercambiador de calor

Se define la eficiencia o coeficiente de desempeño como:

$$
C O P=\frac{Q l}{W}=\frac{Q l}{(Q h-Q l)}=\frac{h_{1}-h_{4}}{h_{2}-h_{1}}
$$

Ql: calor extraído del interior; $W$ : trabajo realizado por el compresor, $Q b$ : calor cedido a los serpentines; $b$ : entalpías

Para poder determinar el COP de la maquina frigorífica, es necesario testear la temperatura y la presión en diferentes etapas del ciclo (entrada y salida compresor, temperatura condensación, temperatura evaporación, entrada y salida de evaporador y condensador). La tabla 1 presenta un resumen de los resultados obtenidos para uno de los ensayos.

\begin{tabular}{|c|c|c|c|}
\hline $\begin{array}{c}\text { Tsal. Evap } \\
\left({ }^{\mathrm{a}} \mathrm{C}\right)\end{array}$ & $\mathbf{1 3}$ & T sobreca $\left({ }^{\mathrm{a}} \mathrm{C}\right)$ & $\mathbf{1 4}$ \\
\hline $\begin{array}{c}\text { T ent. Comp } \\
\left({ }^{\mathrm{a}} \mathrm{C}\right)\end{array}$ & $\mathbf{1 4}$ & $\begin{array}{c}\text { P cond (alta) } \\
(\text { bar })\end{array}$ & $\mathbf{2 3 9 , 5}$ \\
\hline $\begin{array}{c}\text { T sal. Comp } \\
\left({ }^{\mathrm{a}} \mathrm{C}\right)\end{array}$ & $\mathbf{7 1}$ & $\begin{array}{c}\text { T cond } \\
(\text { tabla })\left({ }^{\mathrm{a}} \mathrm{C}\right)\end{array}$ & $\mathbf{4 3}$ \\
\hline T sal. Conden $\left({ }^{\mathrm{a}} \mathrm{C}\right)$ & $\mathbf{4 0}$ & $\mathrm{T}$ ext $\left({ }^{\mathrm{a}} \mathrm{C}\right)$ & $\mathbf{3 2 , 5}$ \\
\hline $\begin{array}{c}\text { P evap (baja) } \\
\text { (bar) }\end{array}$ & $\mathbf{9 4 , 5}$ & & \\
\hline \multicolumn{2}{|c|}{}
\end{tabular}

Tabla 1. Valores medidos de temperaturas $y$ presiones

Una vez obtenidos los parámetros de presión y temperatura, se vuelcan sobre diagramas P-H del refrigerante Freón 22 [7], caracterizando el ciclo, tal como se aprecia en la figura 4 y la Tabla 2 . 
Estados:

1-Ingreso al compresor

2-Descarga del compresor

3-Salida condensador

4-Ingreso al evaporador

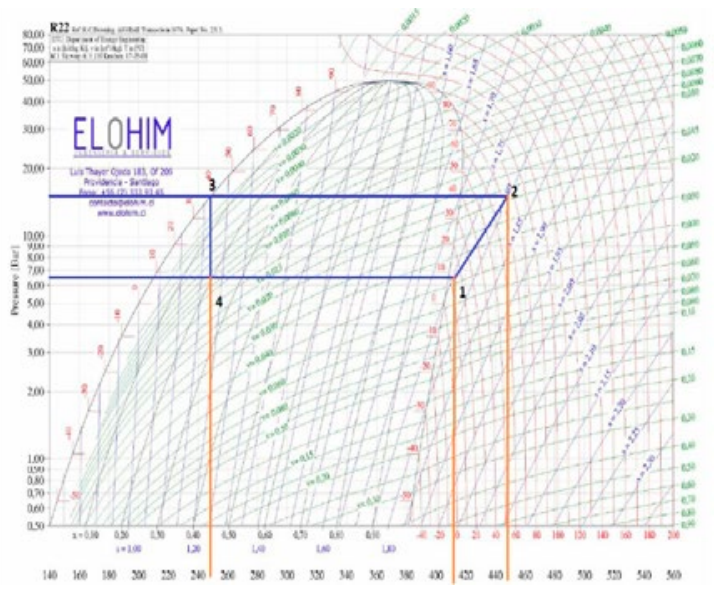

Figura 4: Diagrama P-H de Freón 22

\begin{tabular}{|l|l|l|l|l|l|l|}
\hline $\begin{array}{l}\text { h1 } \\
{[\mathbf{k J} / \mathbf{k g}]}\end{array}$ & $\begin{array}{l}\mathbf{h} 2 \\
{[\mathbf{k J} / \mathrm{kg}]}\end{array}$ & $\begin{array}{l}\mathbf{h} 3=\mathbf{h 4} \\
{[\mathbf{k J} / \mathrm{kg}]}\end{array}$ & $\begin{array}{l}\text { COP } \\
\text { exper }\end{array}$ & $\begin{array}{l}\text { TH } \\
{[\mathbf{K}]}\end{array}$ & $\begin{array}{l}\text { TL } \\
{[\mathbf{K}]}\end{array}$ & $\begin{array}{l}\text { COP } \\
\text { teórico }\end{array}$ \\
\hline 411 & 448 & 249 & 4,3 & 305,5 & 299 & 46 \\
\hline
\end{tabular}

Tabla 2. Entalpias para los estados de operación del ciclo

El rendimiento de una máquina se puede definir de forma general como el cociente entre la energía o el trabajo útil producido por esa máquina, y la energía o el trabajo externo que debe aplicarse a la misma para que produzca el trabajo o el calor que se le demanda.

$$
\operatorname{COP}=\frac{Q_{\text {espacio util }}}{W_{\text {equipo }}}
$$

El calor transferido del espacio útil

Qespacio util=Qsensible aire + Qlatente bumedad

Estos calores se obtienen a partir de la variación de la temperatura y variación de humedad del flujo de aire
Trabajo del equipo $W_{e}=I^{*} V$

Donde: $I$ es la corriente que demanda el compresor en Amper y $V$ es la tensión sobre el mismo.

Se han desarrollado adaptaciones a dos equipos comerciales para implementar, en contextos similares a situaciones reales del futuro campo profesional, en los trabajos prácticos de la asignatura Termodinámica.

\section{CONCLUSIONES}

Un objetivo del equipo docente de la asignatura Termodinámica es la realización de prácticas experimentales, mejorando la calidad de los mismos. Con ese fin se acondicionan instalaciones, se reparan y adaptan equipos. La incorporación de alumnos a estas tareas ha resultado muy satisfactoria como recurso formativo. Estas innovaciones generan interés por la asignatura con un alto grado de motivación y compromiso para el aprendizaje significativo, pues permite desarrollar contenidos conceptuales, procedimentales y actitudinales. 


\section{REFERENCIAS}

\section{[1]. PAOLONI, P Y CHIECHER, A (2013)}

Experiencias de formación y de inserción laboral de ingenieros: las voces de los protagonistas desde una investigación biográfica. Innovación Educativa, ISSN: 1665-2673 vol. 13, número 61, enero-abril.

[2]. Cieza, R; Eirin, M; Muro, M (2012) La formación práctica en la carrera de ingeniería agronómica. el caso del tambo "6 de agosto” IV Congreso Nacional y III Congreso Internacional de Enseñanza de las Ciencias Agropecuarias

[3]. Stoppello, M et all (2016) Promoción del uso de energías no convencionales en clases experimentales de cursos de ingeniería en Facena III Congreso Argentino de Ingeniería IX Congreso Argentino de Enseñanza de la Ingeniería.2016 Libro de Actas pag 808-815 ftp://ftp.frre.utn.edu.ar/libro\%20 de\%20actas\%20cadi-caedi\%202016.pdf

[4]. Práctica L3: Ciclo real simplificado de una máquina frigorífica (2013) https:// alojamientos.uva.es/guia_docente/ uploads/2013/455/42611/1/Documento60. pdf

[5]. Guanipa, G coord. (2010) Práctica ciclo de refrigeración por compresión de vapor https://termoaplicadaunefm.files.wordpress.com/2009/02/practica-2-ciclo-derefrigeracion-por-compresion-de-vapor.pdf [6].https://www.fing.edu.uy/sites/default/files/2012/5682/LETAFT.pdf

[7]. Diagrama de Mollier del R22 http:// elohim.cl/download/r22.pdf

\section{BIBLIOGRAFÍA}

BAROLLI, E et all (2010) Laboratorio didáctico de ciencias: caminos de investigación. Revista Electrónica de Enseñanza de las Ciencias Vol 9, No 1, 88-110.

CASALS TORRENS, P et all (2013) Laboratorios abiertos per el @ prendizaje de Electricidad. Presentació de resultats dels projectes de millora de la docència Institut de Ciències de l'Educació - Universitat Politècnica de Catalunya.

ÇENGEL, Y; BOLES, M. (2006) Termodinámica 6ta Edición. Editorial Mc Graw Hill CONESA, JA (2011) Sistema de refrigeración por compresión https://rua.ua.es/dspace/ bitstream/10045/17271/1/refrigeracion.pdf

MARTÍN, J et all (2009) Equipo Didáctico para Aprendizaje Colaborativo en Automatización e Informática Industrial. Formación Universitaria - Vol. 2 No 5 - 2009. Pág. 31-40.

MIRANDA, A y RUFES, P (2004) Fluidos Frigoríficos. Editorial CEAC técnico climatización

RUFES, P y MIRANDA, A (2004) Ciclos de Refrigeración. Editorial CEAC técnico climatización 